\title{
La fotopolimerización en 2002
}

\author{
Sánchez Soler L.A.*, Espías Gómez A.F.**
}

\section{RESUMEN}

Debido a la reciente aparición de nuevas lámparas de fotopolimerización de alta intensidad y teniendo en cuenta los factores que influyen y los parámetros que determinan la eficacia de la polimerización se discute sobre los distintos tipos de lámparas. Los resultados obtenidos son inferiores en los de alta intensidad (arco de plasma y láser) a los valorados con lámparas halógenas. En cuanto a su evolución las LED son las mejor valoradas por sus ergonómicas ventajas.

Palabras clave: Fotopolimerización, composites fotopolimerizables, lámparas de polimerización.

\begin{abstract}
Due to the recent appearance of new lamps of photopolymerization of high intensity and considering the factors that influence and the parameters that determine the effectiveness of the curing it discusses on the different types from lamps. We've made a review of advantages and disadvantages for each light technology. We explain the main factors wich take importance in these procedures. The obtained results are poorer in those of high intensity (arc of plasma and laser) to the valued ones with halogenous lamps. As far as their evolution the LED could be the right choice because of their ergonomic advantages. We expect a future with total compatibility between dental curing materials and lamps.
\end{abstract}

Key words: Lightcuring, light-cured composites, dental curing lamps.

Aceptado para publicación: octubre 2003.

* Profesor Asociado. Facultad de Odontología. Universidad de Barcelona.

** Profesor Titular. Facultad de Odontología. Universidad de Barcelona.

Sánchez Soler L.A., Espías Gómez A.F. La fotopolimerización en 2002. Av. Odontoestomatol 2004; 20 -6: $289-295$.

El objetivo de esta ponencia es el de discutir el estado actual de la fotopolimerización. Debido a la reciente aparición de nuevas lámparas de fotopolimerización de alta intensidad, consideramos de notable importancia conocer las cualidades de cada una de ellas y su compatibilidad con los materiales restauradores actuales, así como las técnicas recomendadas hoy en día para obtener el máximo rendimiento de las mismas.

\section{INTRODUCCIÓN}

Los materiales restauradores y sistemas adhesivos copan la atención de la mayoría de los profesionales debido a su constante evolución y, quizás, no se dedica el suficiente esfuerzo a la comprensión del instrumento diseñado para polimerizarlos. Consideramos muy útil una puesta al día en este tema, si bien es cierto que todavía se hecha a faltar un mayor número de investigaciones que clarifiquen algunos claroscuros que pueden inducir a la duda.

Los composites están formados por una matriz orgánica con diferentes monómeros (principales, diluyentes, agentes de enlace con el relleno inorgánico, inhibidores de la polimerización, estabilizadores de la radiación ultravioleta, y iniciadores de la polimerización) y un relleno inorgánico que les confiere las propiedades físicas determinantes para ser usados como materiales de restauración. 
Para que se produzca la reacción de polimerización es preciso que el iniciador genere los primeros radicales libres, y para ello debe ser activado por algún agente. Para los composites autopolimerizables el activador será un compuesto químico, mientras que para los fotopolimerizables será la luz con un espectro determinado (1).

\section{FACTORES QUE INTERVIENEN EN LA FOTOPOLIMERIZACIÓN:}

1. Del material
a. Tipo de fotoiniciador
b. Color
c. Grosor de la capa

2. Del foco de luz
a. Longitud de onda
b. Distancia
c. Intensidad
d. Tiempo de exposición

\section{Factores del material}

\section{a. Tipo de fotoiniciador (fig. 1)}

El fotoiniciador más utilizado es la canforoquinona, perteneciente al grupo de las diacetonas. Últimamente, ciertos composites incluyen PPD (1-fenil1,2-propandiona). Como acelerador de la iniciación suelen añadirse aminas las cuales tienen una gran afinidad por los fotoiniciadores.

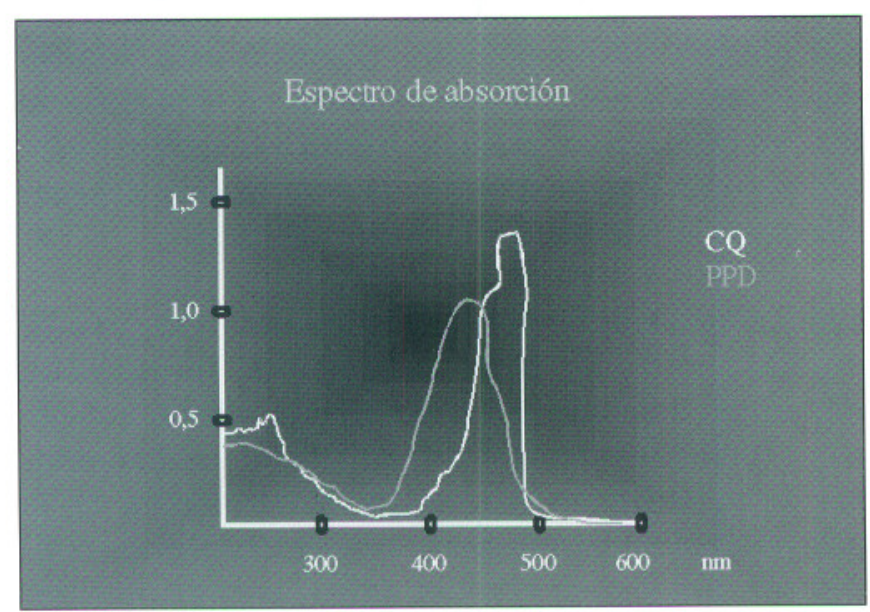

Fig. 1. Espectro de absorción de los fotoiniciadores.
La principal diferencia entre ambos iniciadores radica en el espectro de longitud de onda en el que se activan.

La canforoquinona (CQ) se activa en un rango de entre 400 y $500 \mathrm{~nm}$, siendo su pico de máxima activación los $468 \mathrm{~nm}$.

El PPD se activa entre 400 y $450 \mathrm{~nm}$ y su pico de máxima absorción está en los $410 \mathrm{~nm}$. (2).

\section{b. Color}

Los colores más oscuros contienen pigmentos más opacos que ocasionan fenómenos de dispersión de la luz, por lo que necesitan de un mayor tiempo de aplicación de luz para conseguir una correcta fotopolimerización.

\section{c. Grosor de la capa de composite}

Toda la información de la que disponemos en la actualidad indica que el grosor máximo de cada capa de composite no debe exceder los $2 \mathrm{~mm}$. Este aspecto no está motivado por el grado de polimerización, sino porque a mayor grosor de la capa más contracción de polimerización se producirá, lo que puede ocasionar despegamientos de la capa adhesiva con la correspondiente implicación clínica (3).

\section{Factores del foco de luz}

a. Longitud de onda.- Debería abarcar los picos de máxima activación de los diferentes tipos de fotoiniciadores, para permitirnos utilizar cualquier material restaurador fotopolimerizable.

b. Distancia.- La efectividad de la radiación lumínica es inversamente proporcional al cuadrado de la distancia. Este hecho implica que pequeñas variaciones en la distancia entre el foco de luz y el material implican grandes pérdidas en la intensidad. Por lo tanto la punta de la guía de luz deberá estar lo más próxima a la superficie del material restaurador (4).

c. Intensidad.- La intensidad mínima que debemos exigirle a la lámpara es de $350-400 \mathrm{~mW} / \mathrm{cm}^{2}$. Con la finalidad de realizar las comprobaciones rutinarias resulta de incuestionable interés la tenencia de un radiómetro, que en caso de no venir incorporado a la lámpara, puede adquirirse de forma separada. 
Cualquier descenso de la intensidad por debajo de estos valores nos hará sospechar de un defecto de la bombilla, del filtro, o de la guía de luz (pequeñas fracturas o depósito de restos de composite o minerales por acción del autoclave).

d. Tiempo de exposición.- con las lámparas halógenas actuales debe ser de 40 segundos por capa. Si bien hay composites que se polimerizan bien en 20 segundos, es posible que en ciertos momentos nos separemos de la superficie del material, lo que ocasionaría una disminución de la intensidad efectiva. Si aplicamos la luz 40 segundos por capa estamos compensando este posible suceso.

Es sabido que las lámparas de alta intensidad (plasma, láser, LED) consiguen la polimerización en menos tiempo. No obstante al velocidad de polimerización no es directamente proporcional a la intensidad de la luz. Si doblamos la intensidad de la lámpara, por ejemplo de 400 a $800 \mathrm{~mW} / \mathrm{cm}^{2}$ no disminuiremos el tiempo de polimerización a la mitad, sino que lo haremos un 1,44 más rápido, es decir que pasaremos de necesitar 40 segundos a $400 \mathrm{~mW} / \mathrm{cm}^{2}$ a 27 segundos por capa al doblar la intensidad.

\section{FASES DE LA POLIMERIZACIÓN}

Al fotopolimerizar una resina compuesta se diferencian dos fases:

\section{1. fase pre-gel:}

Presenta un aspecto "gomoso".

Las cadenas formadas tienen una cierta flexibilidad, capaz de absorber las tensiones generadas por la contracción sin transmitirlas a la interfase adhesivodiente (5).

\section{2. fase post-gel:}

En esta fase las cadenas pierden la flexibilidad por lo que ya no son capaces de amortiguar las tensiones generadas las cuales pueden transmitirse a la capa adhesiva con el posible despegamiento puntual de la misma el cual puede originar los problemas clínicos ya conocidos.
Por lo anteriormente expuesto ya podemos intuir la conveniencia de alargar la fase pre-gel $(2,5)$.

\section{Parámetros a evaluar para determinar la eficacia de la fotopolimerización:}

A la hora de comparar el grado de eficacia entre diferentes fuentes lumínicas, lo hacemos a expensas de valorar las cualidades del producto final (composite). A nivel de laboratorio tres son las pruebas fundamentales que se emplean.

\section{Grado de conversión}

Es el porcentaje de monómeros que se transforman en polímeros. Con los materiales y las lámparas actuales se logran valores de hasta un $80-85 \%$.

El aumento de la intensidad lumínica produce un mayor grado de conversión, pero no de una forma directamente proporcional. Por lo tanto el doblar la intensidad de la luz no ocasiona el doble de conversión, sino que este aumento es relativamente pequeño.

\section{Contracción de polimerización}

Si bien los composites con alto contenido de relleno inorgánico han conseguido disminuirla, aún sigue siendo uno de los principales inconvenientes de estos materiales, alcanzando valores del 1,5 al 3,5\%. $(6,7)$.

\section{Propiedades mecánicas}

Se evalúan principalmente la dureza, resistencia a la compresión, flexión y tracción, así como el modulo de Young.

Existe documentación que demuestra que un aumento del tiempo de exposición a una menor intensidad proporciona un material con valores óptimos de estas propiedades.

Así pues una disminución de la intensidad de la luz en la fase inicial produce una mayor duración de la fase pre-gel y por lo tanto la formación de cadena poliméricas de mayor longitud con la consiguiente absorción de tensiones y la optimización de ciertas propiedades mecánicas (8).

Tipos de fotopolimerización:

A. Continua: aplicación de una intensidad de luz constante durante el tiempo de aplicación. 


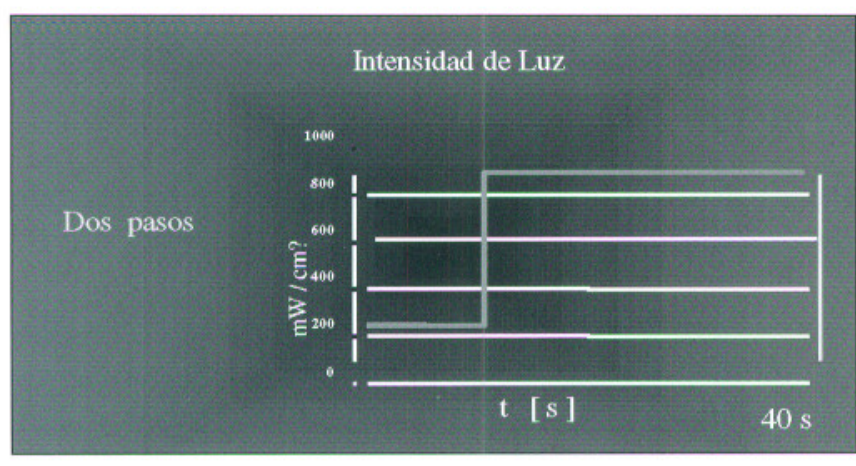

Fig. 2. Intensidad de luz. Dos pasos.

B. Discontinua: Variación de la intensidad en función del tiempo. Dentro de este tipo las más utilizadas son las técnicas de fotopolimerización inicial a baja intensidad, ya que son las que parece ser que mejoran las propiedades del composite. Son tres la técnicas empleadas:

1. fotopolimerización en dos pasos (5) $1^{\text {a }}$ fase a baja intensidad $\left(100-250 \mathrm{~mW} / \mathrm{cm}^{2}\right)$. $2^{\mathrm{a}}$ fase a alta intensidad $\left(500-900 \mathrm{~mW} / \mathrm{cm}^{2}\right)$.

2. fotopolimerización progresiva o "en rampa" Es como la anterior pero la elevación de la intensidad está programada en la lámpara y es progresiva.

\section{3. fotopolimerización diferida (7)}

- Primeros incrementos a $600 \mathrm{~mW} / \mathrm{cm}^{2}$ durante $10 "$

- Última capa a nivel de esmalte a $200 \mathrm{~mW} / \mathrm{cm}^{2}$ durante 3"

- Modelado y pulido

- A los 3-5' se aplica a $600 \mathrm{~mW} / \mathrm{cm}^{2}$ durante 10 " por vestibular, por lingual y por oclusal

Se consigue un endurecimiento de las capas superficiales con alargamiento de la fase pre-gel de las capas más profundas.

\section{TIPOS DE FUENTES LUMÍNICAS}

Debido a la reciente aparición en el mercado de lámparas de alta intensidad, existen diferentes opciones a la hora de adquirir una fuente de fotopolimerización. Creemos que el conocimiento de las principa-

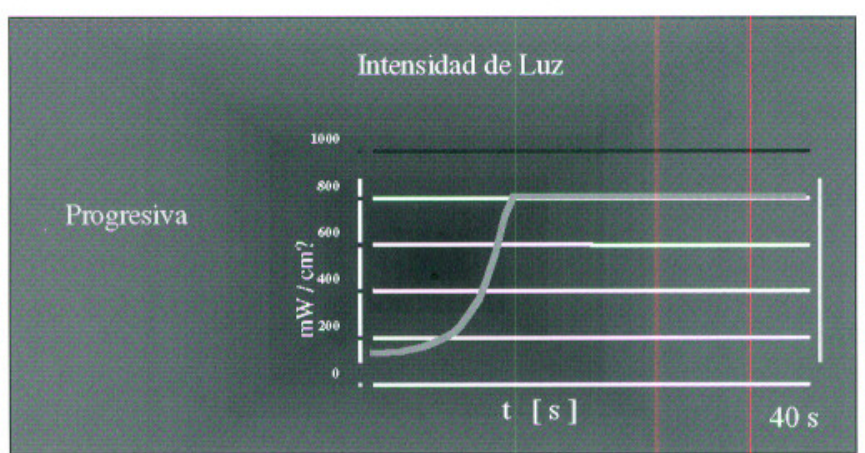

Fig. 3. Intesidad de luz. Progresiva.

les características y su compatibilidad con los materiales actuales resulta de gran importancia.

Podemos diferenciar 4 tipos de lámparas:

1. Lámparas halógenas.

2. Lámparas de arco de plasma.

3. Lámparas de láser.

4. Lámparas de diodos emisores de luz.

1. Lámparas halógenas. Debemos diferenciar aquellas tradicionales de las de nueva generación con rangos de intensidad variables y posibilidad de regulación de la misma.

Tanto unas como otras tienen un espectro de longitud de onda que abarca tanto la canforoquinona como el PPD. Las más modernas con radiómetro incorporado nos permiten seleccionar la intensidad de la luz deseada entre unos niveles, además tienen memorizada una fotopolimerización progresiva, por lo tanto permiten realizar todas las técnicas de fotopolimerización inicial de baja intensidad y su precio es asequible. En contrapartida, las bombillas tienen una vida útil relativamente breve, desprenden bastante calor por lo que se precisa de un ventilador ruidoso y la potencia aprovechada es tan sólo de un 0’7\%.

2. Lámparas de arco de plasma. La bombilla contiene un gas de argón o xenón (fundamentalmente este último) y dos electrodos próximos entre sí. Al aplicar una corriente eléctrica se produce la ionización del gas con la correspondiente emisión de luz. Las características principales de estas lámparas son:

Longitud de onda. 430-500 nm (no polimerizan PPD) 
Intensidad > $1900 \mathrm{~mW} / \mathrm{cm}^{2}$. El tiempo mínimo aceptado actualmente por cada capa de composite es de 10 segundos.

Existen trabajos que demuestran que los composites polimerizados 10 segundos por capa con este tipo de lámparas tienen peores propiedades mecánicas $(9,10)$ y mayor contracción de polimerización (11) que los polimerizados 40 segundos por capa con lámparas halógenas. Por el contrario no se observan diferencias en la eficacia del cementado de brackets al polimerizar 2, 5 o 10 segundos con lámparas de arco de plasma y al hacerlo 20 ó 40 segundos con halógenas (12). Por lo tanto las únicas ventajas constatables de las PAC son el ahorro de tiempo en Ortodoncia y en ciertas técnicas de blanqueamiento (BB.)

3. Lámparas de láser. El láser más empleado para esta finalidad es el láser de argón. Las marcas más conocidas son el ILT Brite Smile y el Laser Med ACU Cure 3000.

La longitud de onda emitida es de 476,5-480 nm y una emisión de energía de alta intensidad (800 $\mathrm{mW} / \mathrm{cm}^{2}$ ). Si bien el hecho de tener un espectro tan estrecho tiene la ventaja de un mayor aprovechamiento de la energía para el pico de la canforoquinona, podemos percatarnos que no polimerizarán correctamente aquellos composites con PPD en su composición. Según la bibliografía consultada se observan discrepancia de resultados al compararlas con las halógenas; de forma que se encuentran propiedades mecánicas inferiores en los composites polimerizados con estas lámparas $(13,14)$ y ciertos autores observan una menor contracción de polimerización cuando se emplean lámparas láser (15), si bien insistimos en que estas comparativas son válidas para aquellos composites que no contengan PPD.

Como podemos constatar, con las lámparas de alta intensidad (arco de plasma y láser) los resultados obtenidos son inferiores a los valorados con lámparas halógenas, y todo parece indicar que esto se debería a que la polimerización a alta intensidad se produce a expensas de cadenas cortas.

4. Lámparas de diodos emisores de luz (LED). Son las últimas que han aparecido en el mercado. La luz no se produce por calentamiento de filamento sino por fenómenos mecánico cuánticos entre semiconductores.

Para estas lámparas el grado de conversión de energía oscila entre el 7 y el $27 \%$ en comparación con las halógenas que únicamente aprovechan el 0,7\% de la energía generada por la bombilla.

Una vez más nos encontramos con el inconveniente de que su rango de longitud de onda, que es de 450 a 490 nm, no coge el pico de máxima absorción del PPD. Por lo tanto su uso se limitará a aquellos materiales cuyo fotoiniciador sea la canforoquinona.

Otro inconveniente es que no permiten la selección del nivel de intensidad, por lo que no podremos realizar las técnicas de polimerización inicial a baja intensidad en dos pasos ni diferida. En cambio si que tiene memorizado un programa de polimerización progresiva.

En contrapartida presentan una serie de ventajas como son:

- Tamaño reducido (la mayoría).

- Larga vida de la batería (hasta 10000 horas de emisión de luz).

- Mínima generación de calor (no necesitan ventilador).

- No necesitan filtro.

- La energía emitida se mantiene constante hasta que se agota la batería.

- Al tener un espectro de longitud de onda más reducido necesitan la mitad de energía que las halógenas para saturar el pico de absorción de la canforoquinona.

En la actualidad en el mercado se encuentran las siguientes lámparas de LED:

- Elipar Freelight (3M-Espe).

- GC E light (GC).

- Lux O Max (Akeda).

- Smartlite (Dentsply.

Como conclusión final de las diferentes opciones que se nos presentan parece razonable considerar que hoy por hoy las lámparas halógenas con intensi- 
dad programable (radiómetro incorporado) son las que nos ofrecen mayores garantías a la hora de fotopolimerizar cualquier material, ya que no debemos olvidar que en la mayoría de los casos no disponemos de la información acerca de la composición real de los materiales empleados por lo que no conocemos que tipo de fotoiniciador contienen.

En cuánto a las expectativas de futuro, quizás las LED son las mejor valoradas por sus ergonómicas ventajas, si bien debe resolverse el problema de incompatibilidad con el PPD, ya sea por acuerdo entre los fabricantes de composites o porque la tecnología LED consiga una emisión de luz con el rango de longitud de onda optimizado para cualquier combinación posible de fotoiniciadores.

Por último consideramos de importancia capital establecer una serie de normas para el correcto mantenimiento de cualquier lámpara de fotopolimerización, ya que de poco sirve emplear un buen sistema adhesivo con una correcta técnica de colocación del composite por capas si la aparatología que utilizamos para conseguir su polimerización no rinde a los parámetros deseados debido a un deterioro que nos pasa inadvertido. Cuatro son los puntos que deberemos evaluar:

1. Limpieza de la guía. Utilizaremos un paño suave. En el caso de que se haya adherido composite frotaremos cuidadosamente con una hoja de afeitar o una espátula envueltas en una gasa ligeramente humedecida con alcohol u otras soluciones destinadas a tal efecto.

2. Esterilización. Las guías de luz pueden esterilizarse, convenientemente embolsadas, en autoclave de vapor de agua a $132{ }^{\circ} \mathrm{C}$. No deben emplearse ningún tipo de solución química ni chemiclave. Conviene resaltar que la exposición a ciclos repetidos de esterilización provoca el depósito de compuestos minerales que disminuyen notablemente la intensidad lumínica (hasta un 25\%), de forma que bastan cinco ciclos para que se observe este fenómeno. Para solventarlo deberemos pulir ambos extremos de la guía. Existen productos para esta finalidad.

3. Filtros. Uno de los elementos que debemos revisar en el caso de que el radiómetro detecte un des- censo de la intensidad, es el filtro. En el caso de observar burbujas o grietas, deberemos proceder a su reemplazo.

4. Bombilla. Debe ser transparente, pudiéndose visualizar los filamentos. Hay que evitar el contacto de los dedos con la superficie reflectante.

Para alargar su vida media, es importante mantener el ventilador funcionando hasta que termine el ciclo de enfriamiento.

En los supuesto de ennegrecimiento, superficie lechosa, rotura de filamentos o grieta de la superficie reflectante, cambiaremos la bombilla.

\section{BIBLIOGRAFÍA}

1. F Roth.- "Les composites" Ed. Masson Paris 1991.

2. Thomas P. Keogh.- "Polimerización iniciada mediante luz: claros y oscuros de las nuevas técnicas". Ideas y trabajos odontoestomatológicos 2001; 2 (1): 29-37.

3. Marais JT, Dannheimer MFG, Germishuys PJ et al.- "depth of cure light-cured composite resin with light curing units of different intensity" J Dent assoc South Afr 1997; 52: 403-7.

4. Versluis A, Tantbironj D.- "Theoretical considerations of contractio stress" Compendium 1999 (supl.25); 20: S15-S24-S32.

5. Sakaguchi R, Peters M, Nelson S, et al.- "effects of polymerization contraction in composite restorations” J Den Res 1992; 20: 178-82. 11.

6. Goracci G and cols. "Influence of light intensity and marginal leakage or resin composite restorations". Quintessence Int 1996; 27: 355-62.

7. Kanca III y col.- "pulse activation: reducing resinbased composite contraction. Stresses at the enamel cavosurfaces margins". Am J Den 1999 12: 107-12. 
8. Burgess y col.- "an evaluation of four lightcuring units comparing soft and hard curing". Pract periodontics and Aesthet Dent 1999 11: 125-32.

9. Peutzfeldt A and cols.-"Characterization of resin composites polymerized with plasma arc curing units". Dental Mat 16 (5): 330-6, Sept 2000.

10. Solomon CS.- "Evaluating the efficacy of curing lights". SADJ 54 (8): 357-62. Agosto 1999.

11. Stritikus J.- "An in vitro study of microleakage of oclusal composites..." Jour Cli Ped Dentistry; 24 (3) 221: 7. Spring 2000.
12. Sfondrini MF and cols. " Effects of conventional and high intensity light-curing..." Am Jour Of Orth \& Dentfac Orthopedics; 119 (1): 30-5. Ene 2001.

13. Zrinka T.- "polymerization of composites using pulsed laser..." Eur Jour of Or Sci, 1995.

14. Ravindar.- "physical properties of composites cured..." Am J Den, 1996.

15. Tar C.- "polymerization Shrinkage of ..." Jou Cl Las Med ESurg, 1997. 
